\title{
Generalidades y manejo correcto de la hoja de enfermería
}

\section{General and Correct Management of the Nursing Sheet}

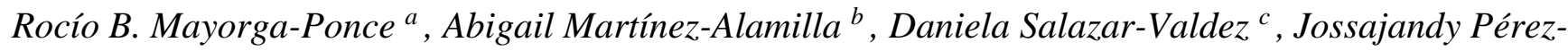 \\ Hernández $^{d}$, Viridiana Ramirez-Gutierrez ${ }^{e}$, Evila Gayosso-Islas $^{f}$
}

\begin{abstract}
:
The nursing sheet is the written evidence of the nursing care and the attention given to the patient, as well as the means of written communication among the nursing professionals Tomas (1996) and Ramos (1990). Nursing records are translated into the part of health care, they are integrated into the nursing sheet. It is necessary scientific rigor, which guarantees the quality of them.
\end{abstract}

\section{Keywords:}

Nursing sheet, registration, patient, communication, document

\section{Resumen:}

La hoja de enfermería es la evidencia escrita de los cuidados de enfermería y atención que es brindada al paciente, además actúa como medio de comunicación escrita entre los profesionales de enfermería Tomas (1996) y Ramos (1990). Los registros de enfermería constituyen una parte fundamental de la asistencia en salud, estando integrados en la hoja de enfermería que forma parte del expediente clínico del paciente, lo que conlleva repercusiones y responsabilidades de índole profesional y legal, que precisa llevarlos a la práctica con el necesario rigor científico, que garantice la calidad de los mismos.

\section{Palabras Clave:}

Seguridad, paciente, lista, verificación, cirugía segura

\section{Introducción}

El registro clínico de enfermería es un documento éticolegal que refleja la función de enfermería en el cuidado, pues recaba la información sobre el estado de salud del paciente durante su estancia hospitalaria. Al considerar lo anterior resulta prioritario que tanto las hojas de enfermería como los registros que el personal realiza en ellas sean adecuados y que revelen la efectividad del cuidado proporcionado.

Por otra parte, los formatos que conforman el expediente clínico incluida la hoja de enfermería han evolucionado en respuesta a la actualización de las legislaciones sanitarias y a la demanda de atención a los servicios de salud, obligando a las instituciones a reestructurar sus formatos con el objeto de facilitar su llenado, evidenciar la productividad y reflejar de manera objetiva la aplicación del proceso de enfermería percibido como instrumento científico de trabajo.

El acto de registrar la información en dicha hoja de manera detallada permite mantener una secuencia en el tratamiento, estancia, cuidado; Siendo una herramienta básica y esencial para los profesionales de enfermería, debe estar orientada a documentar de forma permanente los cuidados en un marco ético legal, ser llenada de una manera correcta además se considera un indicador para la calidad del cuidado. ${ }^{[5]}$

El buen registro es útil para el paciente, porque apoya, mantiene y mejora la calidad de la atención clínica, que necesita una buena fuente de información del estado del paciente. Es una fuente de evaluación para la toma de

\footnotetext{
a Autor de Correspondencia, Universidad Autónoma del Estado de Hidalgo, Instituto de Ciencias de la Salud, Email: robel62@hotmail.com Autor Principal: Universidad Autónoma del Estado de Hidalgo, Instituto de Ciencias de la Salud, abigailmartinez5809@ gmail.com

'Universidad Autónoma del Estado de Hidalgo, Instituto de Ciencias de la Salud, Email: Danielasalazar457@gmail.com

${ }^{\mathrm{d}}$ Universidad Autónoma del Estado de Hidalgo, Instituto de Ciencias de la Salud, Email: jossajandyperez@gmail.com

${ }^{e}$ Universidad Autónoma del Estado de Hidalgo, Instituto de Ciencias de la Salud, Email: viri_gutierrez16@hotmail.com

f Universidad Autónoma del Estado de Hidalgo, Instituto de Ciencias de la Salud, Email: Evila_gayosso_islas@hotmail.com
} 
decisiones y un documento legal que traduce los actos del equipo de salud y apoya la investigación.

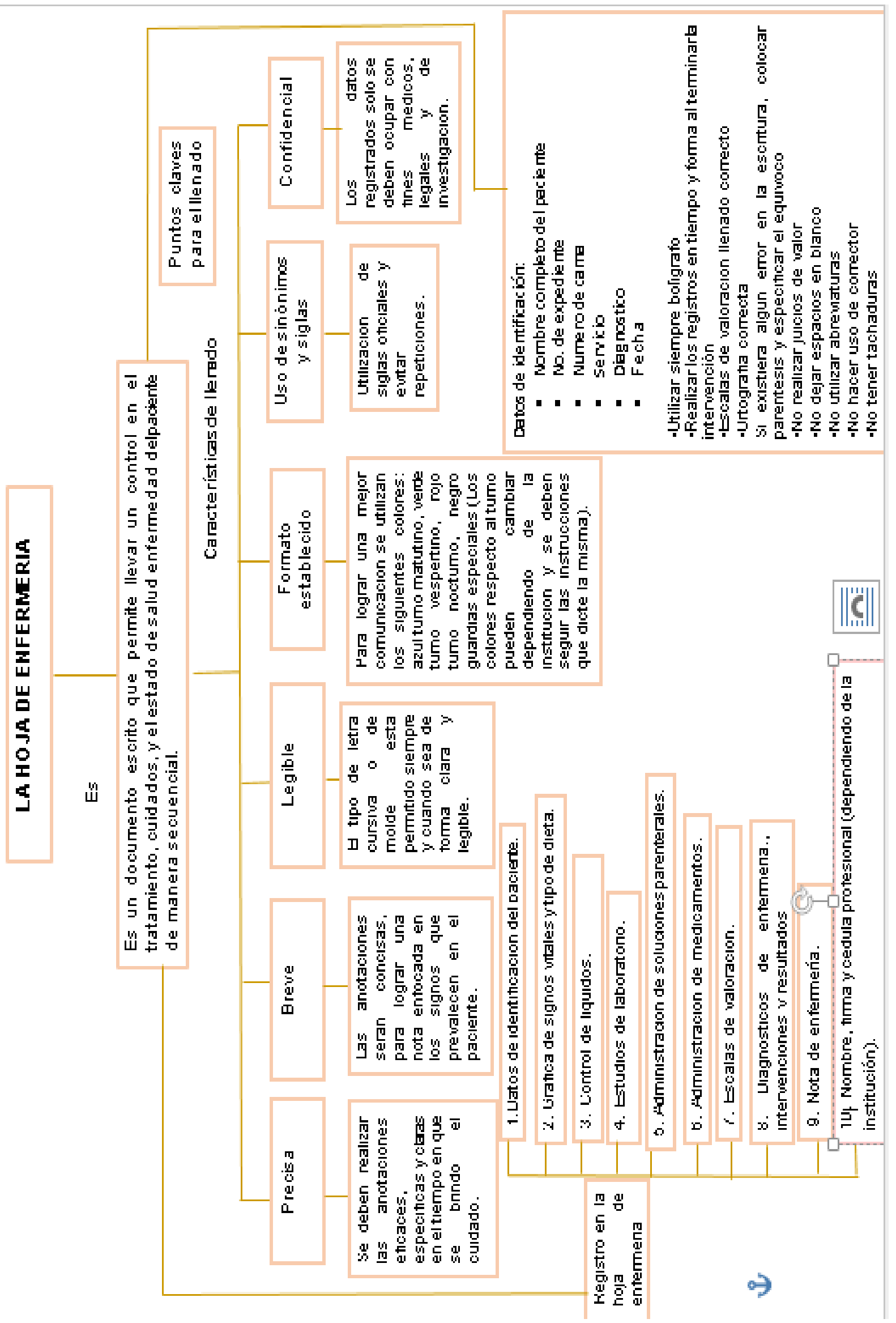




\section{Referencias}

[1] Torres M, Zárate R, Matus R. (2011) Calidad de los registros clínicos de enfermería: Elaboración de un instrumento para su evaluación. [Online]. Revistas UNAM.

[2] Corpus R. (2012) La nota de enfermería como medio de comunicación en la atención del paciente hospitalizado. [Online]. Universidad Autónoma de San Luis Potosí.

[3] Benavent M, Leal M. (2014) Los Registros de Enfermería: Consideraciones ético-legales. [Online]. Bioética.

[4] Secretaría de salud. (2010) NORMA Oficial Mexicana NOM-004SSA3-2012, Del expediente clínico. [Online]. Diario Oficial de la Federación.

[5] Domic E. (2014) Importancia de los registros de enfermería. [Online]. MEDwave.. 